\title{
Proposal for estimating volume based relative abundance of aquatic macrophytes
}

\author{
A. I. Engloner \\ MTA Centre for Ecological Research, Danube Research Institute, Karolina 29, Budapest H-1113, Hungary. \\ E-mail: engloner.attila@okologia.mta.hu,Tel.: +3612793105
}

Keywords: Braun-Blanquet's classes, Kohler-method, Ordinal data, PVI index, Water Framework Directive.

\begin{abstract}
In aquatic macrophyte ecology, species abundance is usually estimated by cover values expressed on the ordinal scale. Recently, there has been increasing demand for three-dimensional estimates of plant abundance. To extend ordinal cover data into three dimensions, a new formula is proposed which considers the vertical developmental types of plants. In this, a constant $k$ is used with three different values reflecting three groups of macrophytes, namely the "free floating leaved"; "rooted, floating leaved" and "submersed leaved" species. By using the new formula, inappropriate conversion and evaluation of ordinal abundance data occurring frequently in the literature may also be avoided.
\end{abstract}

\section{Nomenclature:}

Abbreviations: FFL-Free Floating Leaved, PVI - Plant Volume Inhabited or Plant Volume Infested, RFL-Rooted, Floating Leaved, RPM- Relative Plant Mass, SML-SubMersed Leaved.

\section{Introduction}

Plant abundances are often recorded on multilevel descriptor scales such as Tansley's DAFOR scale (with character states Dominant, Abundant, Frequent, Occasional, and Rare) or the ACFOR scale (Abundant, Common, Frequent, Occasional, and Rare). In aquatic habitats (especially in running waters), a further widespread method is the so-called 'Kohler method' which also estimates the relative species abundance in survey units on a quite similar five-level scale (the character states are: very abundant, abundant, frequent, occasional and rare; Kohler et al. 1971, Kohler 1978). So far, large amounts of macrophyte abundance data have been collected in this way, since international projects such as MIDCC (Multifunctional Integrated Study Danube Corridor and Catchment, started in 2001, see www.midcc.at) facilitated the spread of the method, and the European Standard EN 14184 and national monitoring techniques applied under the regime of the EU Water Framework Directive (Schaumburg et al. 2004, Pall and Moser 2009) accepted this five-level scale for surveying aquatic macrophytes. However, methods relying on scores recorded on the above-mentioned multilevel descriptor scales and the evaluation of such data are burdened by several pitfalls.

These scales are ordinal for which only the relations $<,>$, $=$ and $\neq$ are meaningful mathematically. Since no information is given on the distances between the character states (i.e., we do not know how much more is 'frequent' than 'rare'), evaluation of such data requires either special procedures suitable to the ordinal scale or conversion to the metric scale before any calculations are made.
Nevertheless, the ordinal states are often replaced by numbers $(1=$ rare; 2 = occasional; $3=$ frequent; 4 = abundant; $5=$ very abundant, in the case of Kohler's scale) and involved in arithmetic operations as if they were expressed on the ratio scale. In many cases, the scores have been evaluated by indices, such as the Relative Plant Mass (Kohler and Janauer 1995, Pall and Janauer 1995) and further indices derived from it (see, for instance, Pall and Moser 2009) or by multivariate analyses (for instance Principal Components Analysis and Detrended Correspondence Analysis, see Engloner 2012). In these evaluations, the descriptor states are used as real numbers from 1-5 implying conversion of the original ordinal scores. This has remarkable effects on data structure (see below).

Further difficulties may arise due to the three dimensional development of aquatic vegetation. The most frequently used formula to assess the vertical extension of aquatic macrophytes is PVI (plant volume inhabited or plant volume infested) which is calculated from mean plant cover in percentage of the total observed area (PVI $=$ mean cover $\times$ mean plant height / water depth (Søndergaard et al. 2010)). Although the rare/frequent type values can be converted to percentage data (as discussed later) and, therefore, they could be involved into PVI, a major disadvantage of this calculation is that PVI disregards the morphological variation of plants. Consequently, it cannot be applied to, for example, water lily-like macrophytes which cover much larger space on the surface than underwater.

Another commonly used technique is to raise the ordinal scores to the third power with the purpose of "taking into account the 3D development" of aquatic plants (Janauer 2003, 
Janauer and Heindl 1998). Actually, the third power function is the most widely used conversion of Kohler's scores. However, in addition to the unsuitable multiplication of ordinal data, in many studies it remained unclear whether the descriptor scale represented 2D cover estimates or 3D abundances of species. If the values are meant to incorporate the 3D plant development, the $x^{3}$ function (to involve vertical plant mass) is unnecessary. Otherwise, raising Kohler's scores of all species to the third power is misleading, since the vertical extension of aquatic plants may differ considerably by species (cf. Engloner 2012).

When the values incorporate vertical plant mass, "only" an appropriate conversion method must be chosen. As Engloner (2012) demonstrated, cubic conversion considerably emphasizes high abundance scores, while the use of the $1-5$ values as numbers gives much weight to the frequency of species. If ordinal scores correspond with percentage limits, substitution by the mean values of percentage classes may also be possible. Unfortunately, such classes are not defined for the character states on the Kohler scale. Therefore, Engloner (2012) suggested substitution by the mean values of Braun-Blanquet's (1964) percentage classes and demonstrated that the large ordinal scores and the frequency of species are both taken into consideration in this way. (Although Braun-Blanquet's classes have been developed to estimate area-based plant abundance, the provided percentage limits can also refer to the volume of the water body occupied by a certain plant species. Of course, when the scores of a fivelevel scale are substituted, the lowest two values of BraunBlanquet's scale, i.e., "1" and "+" have to be merged.) The utility of these classes was demonstrated by Engloner et al. (2013) evaluating macrophyte abundance data recorded from the main channel of the Danube River.

However, when sampling provides plant cover values (i.e., only area-based abundance data are available) but, for any reason, estimation of $3 \mathrm{D}$ macrophyte abundance is required, there is no appropriate conversion procedure. Therefore, this paper proposes a new formula which considers the developmental differences of macrophytes and avoids the disadvantages of inappropriate data conversions.

\section{Estimation of volume based abundance}

The new method projects vertically the area based plant abundance such that the three dimensional extension of aquatic macrophytes is considered. For this purpose, three morphological categories are distinguished based on the proportion of plant organs occurring in the water body. The first is the group of non-rooted, free floating leaved species which, compared to the species of the further two groups, do not penetrate into the water body considerably (such as Lemna, Spirodela and Salvinia species); hereafter this group is referred to as "free floating leaved" (FFL) plants. Rooting species attached to the bottom and having most or all leaves floating on the water surface (for instance Nymphaea, Nuphar and Trapa species) are in the second group; "rooted, floating leaved" (RFL) species. Submersed macrophytes whose all (or nearly all) organs are under the water surface (for example,

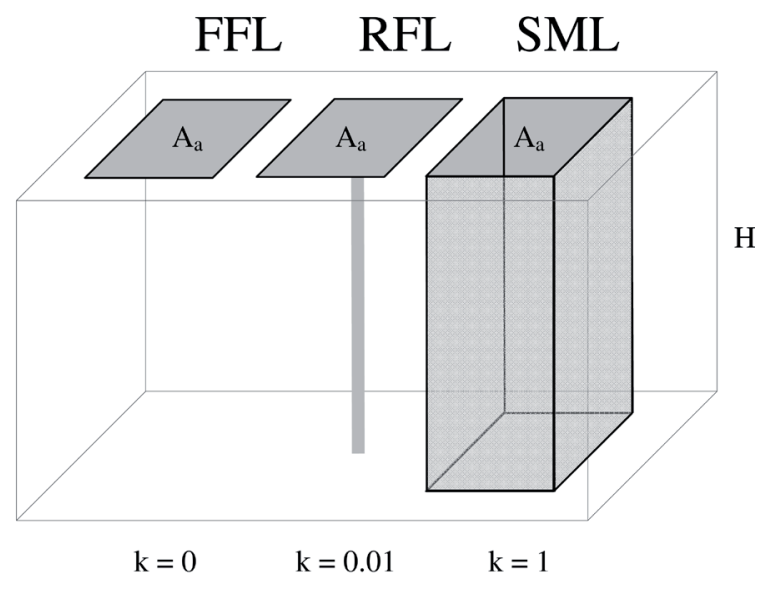

Figure 1. Projection of area based abundances (Aa) of macrophytes differing in vertical development. Morphological categories are: FFL - free floating leaved, RFL - rooted, floating leaved and SML - submersed leaved plants; $\mathrm{H}$ : height of water column; k: constant values depending on the vertical extension of species.

Ceratophyllum, Myriophyllum and Najas species) form the third category; the "submersed leaved" (SML) plants.

Based on these morphological categories, a constant $k$ with three different values is introduced in order to implement the plant developmental discrepancies in the vertical projection of area based abundances. The values of $k$ are chosen to be $0,0.01$ and 1 for FFL, RFL and SML species, respectively. Two of the three values are obvious: 0 provides no vertical projection for species which have negligible vertical development, while the total 2D extension is projected vertically when $k=1$ (in the case of SML macrophytes). RFL species, however, have various organs (with various extensions) floating on the water surface (e.g., leaves or rosettes) and running towards the bottom (e.g., bare petioles of Nymphaea and Nuphar species or stems with alternating root-like submerged leaves of Trapa). Therefore, the suggested value of 0.01 for RFL plants is only a rough estimate of the ratio between the area of the floating and the underwater organs. (Actually, this $k$ value cannot be determined precisely, since this ratio highly varies from species to species and from individuals to individuals.) Nevertheless, the new method was tested with various $k$ values $(0.01,0.1$ and 0.2$)$ applied to RFL plants and the results were little influenced.

Volume based abundances are then calculated by the following equation:

$$
A_{v}=A_{a}+A_{a} \cdot H \cdot k
$$

where $A_{v}$ and $A_{a}$ are the volume and area based abundance values, $H$ is the height of the water column at the survey unit (in centimeter) and $k$ is the constant depending on the three morphological categories. If submersed macrophytes do not reach the water surface (or they grow above the surface), $H$ refers to the actual height of the plants. 


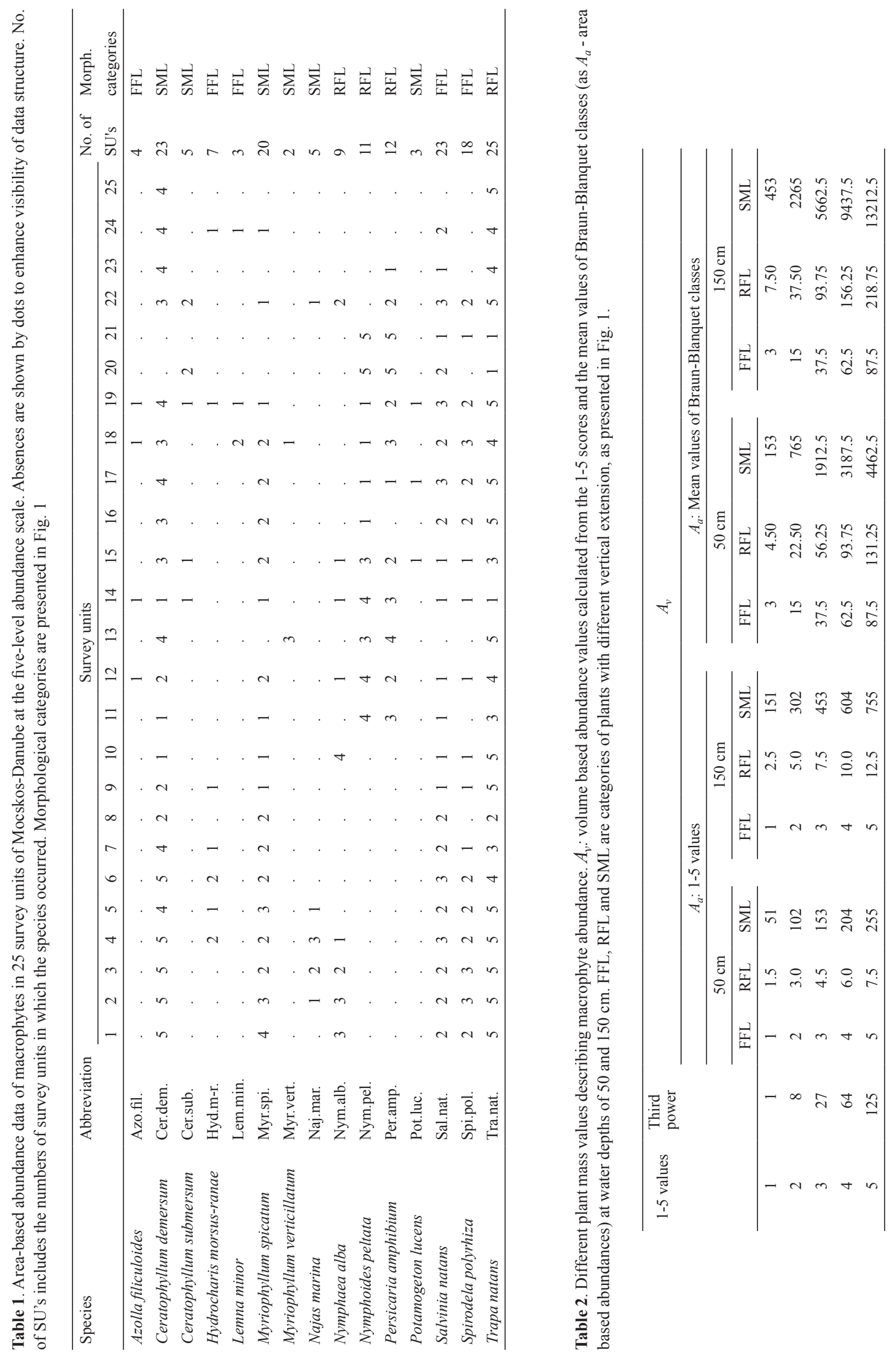


The formula can be rewritten as

$$
A_{v}=(1+k \cdot H) \cdot A_{a}
$$

which indicates more clearly that $A_{v}$ is obtained from $A_{a}$ weighted by $H$ and $k$.

Using this formula, area based abundances are transformed on the basis of the three different vertical extension types of plant masses shown in Fig. 1.

\section{Evaluation of an illustrative data set}

For illustration, the new method is applied here to abundance data (Table 1) recorded in 2012 at the MocskosDanube (a former Danube side branch lying on the unprotected area of the Béda-Karapancsa Landscape Protection Area, Hungary) according the Kohler method. The ordinal descriptor states were used to estimate the relative species cover and were converted to the metric scale after Engloner (2012). Therefore, $A_{a}$ values involved in the proposed equation were (i) the original scores as 1-5 numbers and (ii) the mean values of Braun-Blanquet's classes (where plant covers between 0 and $5 \%$ were merged into one class): $3(0<x \leq$ $5 \%) ; 15(5<x \leq 25 \%) ; 37.5(25<x \leq 50 \%) ; 62.5(50<x \leq$ $75 \%)$ and $87.5(75<x \leq 100 \%)$.

For simplicity, uniform water depth was used for all survey units and, to demonstrate the effect of different water depths on the 3D transformation, volume based abundances were calculated with depths of 50 and $150 \mathrm{~cm}$.

After calculating the $A_{v}$ values, the dominance order of species was determined by using the Relative Plant Mass (RPM) index, which is the percentage of relative plant mass of each species, weighted by the length of survey units

$$
\mathrm{RPM}_{h}[\%]=\frac{\sum_{i=1}^{n}\left(\mathrm{PM}_{h i} \cdot L_{i}\right) \cdot 100}{\sum_{j=1}^{k}\left(\sum_{i=1}^{n}\left(\mathrm{PM}_{j i} \cdot L_{i}\right)\right.}
$$

where $\mathrm{RPM}_{h}$ is the relative plant mass of species $h$; $\mathrm{PM}_{h i}$ is the plant mass of species $h$ in survey unit $i$ and $L_{i}$ is the length of survey unit $i$ (Pall and Janauer 1995). In our case, $\mathrm{PM}_{h i}$ is the volume based abundance $\left(A_{v}\right)$ of a certain species.

For comparison, the 1-5 values of Kohler's descriptor scale and their cubic conversion (i.e., the two frequently used estimates representing 2D or 3D abundance of species, as mentioned above) were also evaluated. All plant mass data involved in the RPM index are presented in Table 2.

\section{Changes in species dominance order}

Based on the RPM percentages, Table 3 presents how the species dominance orders changed when different plant mass values were assessed by the index (high RPM\% means high relative species dominance). When 1-5 and cubic values were used, Trapa natans (high abundance values recorded in 25 survey units, see Table 1) was the first and Ceratophyllum demersum (high abundance values from 23 locations) the second species in the dominance order. Considering the other species, macrophytes with high occurrence numbers (high frequencies) were taken greatly into account when numbers 1-5 were used, while species with high abundances were emphasized based on the third power data. For instance, Salvinia natans (low abundance scores from 23 survey units) preceded Nymphoides peltata (mostly high abundance in 11 habitats) in the first case, with the reverse ordering in the second case.

Highly different results were obtained with volume based abundances calculated by the newly proposed equation. Irrespectively of the types of $A_{a}$ values (i.e., 1-5 scores or the mean values of Braun-Blanquet's classes) and the water depths involved into the calculation, the submersed Ceratophyllum demersum, Myriophyllum spicatum and Najas marina were the three most dominant species. In contrast to their high previous ranks in species ordering, the "rooted, floating leaved" Trapa became the $7^{\text {th }}$ and the $4-6^{\text {th }}$, while the "free floating leaved" Salvinia the 10 $0^{\text {th }}$ and $11^{\text {th }}$ (based on the 1-5 values and the mean values of Braun-Blanquet's classes). In general, submerged (SML) species were moved forward and free floating (FFL) macrophytes backward.

Table 3 also demonstrates the effect of the three times higher water depth on plant dominance. The species orders were almost identical when values 1-5 were used in the new formula; regardless of whether 50 or $150 \mathrm{~cm}$ water depth was considered (only Nymphaea alba moved two positions forward in the deeper water). Species orders were also very similar when the mean values of Braun-Blanquet's classes provided the $A_{a}$ scores. However, in this case three submerged species (namely Myriophyllum verticillatum, Ceratophyllum submersum and Potamogeton lucens) were more dominant at a depth of $150 \mathrm{~cm}$ than at $50 \mathrm{~cm}$.

The proportions between the $A_{v}$ values within the morphological (FFL, RFL and SML) categories are equal and are exactly the same as those between the area based values substituting the original ordinal statements. When the 1-5 conversion was applied, the ratio between 'very abundant' and 'rare' is 5 in all cases; 5, 7.5, 255, 12.5 and 755 are five times more than 1, 1.5, 51, 2.5 and 151, respectively (see Table 2). When $A_{a}$ data were converted after Braun-Blanquet (1964), the ratio (29.17) was also unchanged $(87.5 / 3=131.25 / 4.5=$ $4462.5 / 153=218.75 / 7.5=13212.5 / 453=29.17)$.

\section{Discussion and conclusions}

Ordinal data (regardless of whether they represent area or volume based plant abundance) require suitable mathematical methods which, however, reduce ordinal information to presence/absence data, so information is lost (cf. Podani 2005, 2006, Engloner 2012). If the aim is to evaluate these ordinal scores by arithmetic operations or metric multivariate methods, conversion to the metric scale is inevitable. Actually, if those methods (or just a simple subtraction or multiplication) are applied to the original scores, conversion "happens automatically": the values which were only the easy to use replacements of the descriptor states become 
Table 3. Dominance orders based on RPM\% calculated after the 1-5 and third power conversions and from different $A_{v}$ values. The first column under each method shows the species (full names are given in Table 1), while the second columns present the RPM\%. $A_{v}$ : volume based abundance values calculated from the 1-5 scores and the mean values of Braun-Blanquet classes (as $A_{a}$ - area based abundances) at water depths of 50 and $150 \mathrm{~cm}$.

\begin{tabular}{|c|c|c|c|c|c|c|c|c|c|c|c|}
\hline \multirow{3}{*}{\multicolumn{2}{|c|}{$1-5$ values }} & \multirow{3}{*}{\multicolumn{2}{|c|}{ Third power }} & \multicolumn{8}{|c|}{$A_{v}$} \\
\hline & & & & \multicolumn{4}{|c|}{$A_{a}: 1-5$ values } & \multicolumn{4}{|c|}{$\begin{array}{c}A_{a}: \text { Mean values of Braun-Blanquet } \\
\text { classes }\end{array}$} \\
\hline & & & & \multicolumn{2}{|c|}{$50 \mathrm{~cm}$} & \multicolumn{2}{|c|}{$150 \mathrm{~cm}$} & \multicolumn{2}{|c|}{$50 \mathrm{~cm}$} & \multicolumn{2}{|c|}{$150 \mathrm{~cm}$} \\
\hline Tra.nat. & 24 & Tra.nat. & 39 & Cer.dem. & 54 & Cer.dem. & 55 & Cer.dem. & 68 & Cer.dem. & 69 \\
\hline Cer.dem. & 19 & Cer.dem. & 24 & Myr.spi. & 26 & Myr.spi. & 26 & Myr.spi. & 18 & Myr.spi. & 19 \\
\hline Sal.nat. & 10 & Nym.pel. & 11 & Naj.mar. & 5.6 & Naj.mar. & 6 & Naj.mar. & 3.7 & Naj.mar. & 3.7 \\
\hline Myr.spi. & 9 & Per.amp. & 9 & Cer.sub. & 4.9 & Cer.sub. & 5 & Tra.nat. & 2.8 & Myr.vert. & 2.5 \\
\hline Per.amp. & 8 & Sal.nat. & 4.5 & Myr.vert. & 2.8 & Myr.vert. & 2.8 & Myr.vert. & 2.4 & Cer.sub. & 2.4 \\
\hline Nym.pel. & 7.8 & Myr.spi. & 4.3 & Pot.luc. & 2.1 & Pot.luc. & 2.1 & Cer.sub. & 2.3 & Tra.nat. & 1.6 \\
\hline Spi.pol. & 7.8 & Spi.pol. & 3.2 & Tra.nat. & 2.0 & Tra.nat. & 1.2 & Nym.pel. & 0.79 & Pot.luc. & 0.55 \\
\hline Nym.alb. & 4.4 & Nym.alb. & 2.9 & Per.amp. & 0.67 & Per.amp. & 0.39 & Per.amp. & 0.73 & Nym.pel. & 0.45 \\
\hline Hyd.m-r. & 2.2 & Naj.mar. & 0.8 & Nym.pel. & 0.65 & Nym.pel. & 0.38 & Pot.luc. & 0.54 & Per.amp. & 0.42 \\
\hline Naj.mar. & 1.9 & Myr.vert. & 0.6 & Sal.nat. & 0.59 & Nym.alb. & 0.21 & Nym.alb. & 0.31 & Nym.alb. & 0.18 \\
\hline Cer.sub. & 1.7 & Hyd.m-r. & 0.42 & Spi.pol. & 0.44 & Sal.nat. & 0.20 & Sal.nat. & 0.05 & Sal.nat. & 0.02 \\
\hline Azo.fil. & 1.0 & Cer.sub. & 0.40 & Nym.alb. & 0.37 & Spi.pol. & 0.15 & Spi.pol. & 0.04 & Spi.pol. & 0.01 \\
\hline Lem.min. & 1.0 & Lem.min. & 0.19 & Hyd.m-r. & 0.12 & Hyd.m-r. & 0.04 & Hyd.m-r. & 0.01 & Hyd.m-r. & 0.004 \\
\hline Myr.vert. & 1.0 & Azo.fil. & 0.08 & Azo.fil. & 0.05 & Azo.fil. & 0.02 & Azo.fil. & 0.01 & Azo.fil. & 0.002 \\
\hline Pot.luc. & 0.7 & Pot.luc. & 0.06 & Lem.min. & 0.05 & Lem.min. & 0.02 & Lem.min. & 0.01 & $\begin{array}{l}\text { Lem. } \\
\text { min. }\end{array}$ & 0.002 \\
\hline
\end{tabular}

numbers with well-defined differences between each other. The present study demonstrated that the differences between the substituting numbers highly affect the results and determine, for instance, the correlations between the species or their dominance order. The choice of scale for abundances and the procedures converting ordinal statements to the metric scale is always up to the investigator. However, the use of Braun-Blanquet's classes seems to have some advantages. First, Braun-Blanquet's ordinal scores correspond with percentage limits, therefore they can be easily replaced by the mean values of the classes. Secondly, in contrast to the 1-5 and the $\mathrm{x}^{3}$ conversions, neither the large ordinal scores nor the frequencies of the species are overemphasized when this scale is used.

Further problems may arise when the purpose is to characterize the 3D development of aquatic plants. When only area based abundance data are available, the most widely used method is raising the descriptor scores of all recorded species to the third power (Janauer 2003, Janauer and Heindl 1998). However, neither the highly different 3D structure of aquatic plants, nor the dependence of vertical extension on water depth is taken into account in this way. As it was demonstrated, the rare - frequent type ordinal values can be converted to percentage data which, therefore, could be involved into PVI. However, this formula disregards the morphological variation of plants and can only be applied to submersed macrophytes.

For the above reasons, in this paper the area based plant abundances are projected vertically by using a new formula considering the developmental differences and the vertical extension of plants. Of course, the proposed morphological categories (free floating leaved; rooted, floating leaved and submersed leaved plants) offer only a rough categorization of macrophytes, but this way at least the three basic developmental types of aquatic plants appear in the calculations. Although many peculiarities (for instance, the thickness of plant parts floating on the water surface and the diameter of stems or petioles attached to the bottom) are ignored, the new formula does make distinction between the vertical developments of plants and can be applied to any aquatic macrophytes. Certainly, the $k$ value $(0.01)$ chosen for RFL plants is only a rough estimate of the ratio between the area of the floating and the underwater organs and, if higher $k$ values, for instance, 0.1 or 0.2 are chosen, the calculated volume based abundances increase. Nevertheless, the main developmental differences (i.e., the three morphological categories) are maintained by the vertical projection, despite the few tenths of differences in $k$ values applied to RFL plants.

The $3 \mathrm{D}$ values calculated by the new method depend on water depth (the higher the water depth the larger the submersed macrophytes), however, as it was demonstrated, the method is not very sensitive to this factor. The differences between $A_{v}$ values (derived from the same area based abundances) were mostly caused by $k$ (i.e., by the morphological categories), while a three times higher water depth value (150 compared to $50 \mathrm{~cm}$ ) had little effect on plant dominance order. It must be emphasized that if submersed macrophytes do not reach the water surface (or they grow over the surface), the "height values" involved into the new equation refer to the actual height of the stands.

The volume based abundances calculated by the new formula keep the initial proportions between the converted cover 
data and, at the same time, incorporate the developmental differences of plants. Based on these $A_{v}$ values, any arithmetic operations and metric multivariate analyses can be performed appropriately.

Theoretically, PVI could be modified to be able to consider the morphological variance of plants. On one hand, in the equation of PVI, the "mean plant height" can also refer to the actual height (or thickness) of non-rooted, free floating leaved plant material. On the other hand, the equation can be modified for RFL plants as follows: PVI $=$ mean cover $\times$ water depth $\times$ the area ratio of the floating and the underwater organs. However, PVI expresses, by definition, the volume of plants; therefore, the actual plant height (thickness) and the above mentioned ratio have to be determined precisely for each species. This is impracticable, especially for the latter. If an estimate substitutes the ratio between the area of the floating and the underwater organs (as it happens with the use of the introduced constant $k$ ), we almost get back the newly proposed formula.

The new equation, however, does not intend to give the real volume of plant mass, but to provide volume based relative abundance of any aquatic macrophytes (not merely the submersed ones), therefore it can be applied to quite large survey units (for instance, to one-kilometer-long river sections). The proposed method is suitable not only to the evaluation of existing data bases (for this, certainly, the presence of water depth or plant height data is prerequisite) but also to new field assessments. (Of course, during new field surveys, the area based abundance can be recorded not only on ordinal scales, but it can be expressed directly as mean plant cover in percentage and, therefore, no additional conversion is needed before data evaluation.) Finally, the proposed volume based relative abundance values can be involved easily into the sophisticated indices of assessment systems elaborated according to the requirements of the EU Water Framework Directive (Schaumburg et al. 2004, Pall and Moser 2009).

Acknowledgements: This work was supported by the Hungarian Scientific Research Grant (OTKA K106177) and the János Bolyai Research Scholarship of the Hungarian Academy of Sciences.

\section{References}

Braun-Blanquet, J. 1964. Pflanzensoziologie. Springer Verlag, Wien, New York.
Engloner, A.I. 2012. Alternative ways to use and evaluate Kohler's ordinal scale to assess aquatic macrophyte abundance. Ecol. Ind. 20: $238-243$.

Engloner, A.I., E. Szalma, K. Sipos and M. Dinka. 2013. Occurrence and habitat preference of aquatic macrophytes in a large river channel. Community Ecol. 14: 243-248.

European Standard EN 14184 (2003). Water quality-guidance standard for the surveying of aquatic macrophytes in running waters. Comite' Europe'enne de Normalisation, Brussels.

Janauer, G.A. 2003. Methods. Arch. Hydrobiol. Suppl. 147 (Large Rivers, 14): 9-16.

Janauer, G.A. and E. Heindl. 1998. Die Schatzskala nach Kohler: Zur Gültigkeit der Funktion $\mathrm{f}(\mathrm{x})=\mathrm{ax} 3$ als Mas für die Pflanzenmenge von Makrophyten. Verh. Zool.-Bot. Ges. Österreich. 135: 117128.

Kohler, A. 1978. Methoden der Kartierung von Flora und vegetation von Süßwasserbiotopen. Landschaft Stadt. 10: 73-85.

Kohler, A. and G.A. Janauer. 1995. Zur Methodik der Untersuchung von aquatischen Makrophyten in Fließgewassern. In C. Steinberg, H. Bernhardt and H. Klapper (eds.), Handbuch Angewandte Limnologie. Ecomed Verlag, Landsberg/Lech. pp. $3-22$.

Kohler, A., H. Vollrath and E. Beisl. 1971. Zur Verbreitung, Vergesellschaftung und Ökologie der Gefaß-Makrophyten im Fließ wassersystem Moosach (Münchener Ebene). Arch. Hydrobiol. 69: 333-356.

Pall, K. and G.A. Janauer. 1995. Die Makrophytenvegetation von FIusstauen am Beispiel der Donau zwischen FIus-km 2552,0 und 2511,8 in der Bundesrepublik Deutschland. Arch. Hydrobiol. Suppl. 101 (Large Rivers, 9): 91-109.

Pall, K. and V. Moser. 2009. Austrian Index Macrophytes (AIMModule 1) for lakes: a Water Framework Directive compilant assessment system for lakes using aquatic macrophytes. Hydrobiologia 633: 83-104.

Podani, J. 2005. Multivariate exploratory analysis of ordinal data in ecology: pitfalls, problems and solutions. J. Veg. Sci. 15: 497510 .

Podani, J. 2006. Braun-Blanquet's legacy and data analysis in vegetation science. J. Veg. Sci. 17: 113-117.

Schaumburg, J., C. Schranz, J. Foerster, A. Gutowski, G. Hofmann, P. Meilinger, S. Schneider and U. Schmedtje. 2004. Ecological classification of macrophytes and phytobenthos for rivers in Germany according to the Water Framework Directive. Limnologica 34: 283-301.

Søndergaard, M., L. S. Johansson, T. L. Lauridsen, T. B. Jørgensen, L. Liboriussen and E. Jeppesen. 2010. Submerged macrophytes as indicators of the ecological quality of lakes. Freshwater Biol. 55: 893-908.

Received August 4, 2014 Revised September 22, 2014 Accepted December 28, 2014 